\title{
COMMENTARX
}

\section{The treatment of acute bacterial sinusitis: No change is good medicine}

\section{Nicole Le Saux MD}

$\infty \quad$ See related article page 845

$\mathrm{T}$ hat nagging head cold! Every clinician has faced the dilemma of deciding whether to "watch and wait" or to treat rhinosinusitis. Previous trials have taught us that the majority of patients with acute viral sinusitis will recover over the course of 7-Io days, and even patients with acute bacterial sinusitis have a $50 \%-70 \%$ chance of recovery without antibiotics. ${ }^{1}$ Most clinicians have accepted this conservative course of action provided that appropriate follow-up is assured. When prolonged symptoms, high fever or significant pain suggest a need to treat, the physician must choose an appropriate antimicrobial. In this issue Karageorgopoulos and colleagues ${ }^{2}$ present the results of their meta-analysis of fluoroquinolones versus the standard $\beta$-lactam antibiotics for the treatment of acute bacterial sinusitis. Do their findings indicate a need for change in our therapeutic approach?

Based on sensitivity studies conducted in the laboratory, newer "respiratory" fluoroquinolones, such as levofloxacin and moxifloxacin, have enhanced activity against Streptococcus pneumoniae compared with ciprofloxacin and are active against Haemophilus influenzae and Moraxella catarrhalis. This range of antibacterial activity makes them potentially useful in the treatment of respiratory tract infections such as sinusitis and pneumonia. Fluoroquinolones also provide excellent coverage against other respiratory pathogens, including Legionella and Mycoplasma, as well as gram-negative bacteria such as Pseudomonas. However, they are not approved for use in children.

In their meta-analysis, Karageorgopoulos and colleagues ${ }^{2}$ included randomized controlled studies in which the diagnosis of acute bacterial sinusitis was based on various clinical criteria, whether or not further supported by radiologic or microbiologic criteria. The primary outcome examined was "clinical success," defined as resolution of signs and symptoms of acute sinusitis and, in some studies, resolution of radiologic findings and pathogen eradication within IO-2I days. This appears to be a rational, practical outcome to which every clinician can relate. Of the IgI articles the authors identified in their initial literature search, only II were retained for analysis, of which only 9 used respiratory fluoroquinolones. Five of the trials were not blinded, which may have led to bias in the analysis of the results. The authors also revealed that, in most of the studies, there was either financial

\section{Key points of the article}

- Most episodes of acute sinusitis resolve without antibiotic therapy.

- Ampicillin or cephalosporins are just as effective as levofloxacin or moxifloxacin in the treatment of acute bacterial sinusitis.

- Fluoroquinolones can rapidly induce resistance in bacteria, especially Streptococcus pneumoniae, which leads to treatment failure. This effect is especially important in patients who received a fluoroquinolone within 3 months before the present treatment.

- Use of fluoroquinolones as first-line therapy for acute sinusitis should be discouraged.

support from pharmaceutical companies associated with the fluoroquinolone agents tested or affiliation of the authors with these companies. This is understandable, given that fluoroquinolones are new, but independent arm's-length studies would ensure that some of the inherent biases often identified in industry-funded trials would be addressed.

When the authors included all of the patients who were eligible and randomly assigned to the treatment arms in their analysis, they found no clinically important or statistically significant difference in the rates of clinical success between patients who received $\beta$-lactam antibiotics (penicillins and cephalosporins) and those given a fluoroquinolone. This lack of difference is no surprise, since both treatment arms probably included patients whose condition would have improved without treatment. If there is no difference in clinical outcome, how should physicians choose an antimicrobial for the treatment of acute bacterial sinusitis? Should we be more concerned about resistance to $\beta$-lactam antibiotics or to the newer fluoroquinolones? Because $S$. pneumoniae remains the most important pathogen for acute bacterial sinusitis, it becomes relevant to compare the potential patterns of resistance of each type of antimicrobial.

Fluoroquinolones kill bacteria by binding to enzymes that are essential for DNA synthesis and maintenance (specifically,

Nicole Le Saux is with the Department of Infectious Diseases, Children's Hospital of Eastern Ontario, Ottawa, Ont. 
topoisomerase IV in gram-positive bacteria and DNA gyrase in gram-negative bacteria). In addition to an efflux mechanism, resistance to fluoroquinolones occurs when there are spontaneous mutations in any of the genes responsible for the production of these enzymes. Also, these mutations occur as a single step and thus may develop rapidly during therapy. A first mutation results in low-level resistance and carries some risk of treatment failure. Since most episodes of acute sinusitis resolve anyway, this low-level resistance may not be important for most patients. However, a subsequent course of treatment with a quinolone could result in a second mutation and highlevel resistance, even across quinolone classes. Clinical failure would be more likely with this level of resistance and has been documented during therapy with ciprofloxacin and levofloxacin for pneumococcal infections. ${ }^{3}$ Indeed, a history of fluoroquinolone use in the previous 3 months would be a relative contraindication for subsequent treatment of a pneumococcal infection with a fluoroquinolone. As such, physicians prescribing these drugs should be aware of the consequences of sequential use of this class of drugs.

So far, resistance of respiratory pathogens to fluoroquinolones has remained low in Canada $(<2 \%$ of isolates tested in 2002) ${ }^{4}$ probably because fluoroquinolones have only recently been the focus of treatment of respiratory tract infections and because these drugs are not routinely used in children. In the United States, rates of fluoroquinolone-resistant isolates are low $(<\mathrm{I} \%)$, in part because of the widespread use of conjugated pneumococcal vaccine, but clonal spread of the resistant serotypes may be emerging. ${ }^{5}$ In Italy, $5.6 \%$ of pneumococcal isolates collected between $200 \mathrm{I}$ and 2004 were resistant to levofloxacin, and there was evidence of clonal dissemination. ${ }^{6}$ Many of these isolates were multidrug resistant.

Risk factors for pneumococcus resistance to levofloxacin include residence in a long-term care facility, the presence of chronic obstructive pulmonary disease, hospital-acquired infection and previous fluoroquinolone use. The broad spectrum of fluoroquinolones means that resistance mutations can also occur in Escherichia coli and other gut flora. Whether in nasal or oral secretions or stool, the pool of bacteria with significant resistance mutations then has the potential to spread to other people who have never received antibiotics.

In contrast, the mechanism of resistance to $\beta$-lactams by $S$. pneumoniae is acquisition of exogenous DNA, which leads to alterations of one of many $\beta$-lactam-binding sites. The alterations occur gradually, and the level of resistance depends on the number of targets modified. Contrary to fluoroquinolone resistance, resistance to $\beta$-lactam antibiotics does not occur during therapy and is not spontaneous. Furthermore, when treating sinusitis or pneumonia, this resistance can often be overcome pharmacokinetically by increasing the doses and frequency of the $\beta$-lactam antibiotic in order to achieve killing levels of the drug at the site of infection for an adequate amount of time. However, the doses of amoxicillin in the trials reviewed by Karageorgopoulos and colleagues would be considered either too low (e.g., 20-25 mg/kg daily versus recommendations for at least $40 \mathrm{mg} / \mathrm{kg}$ daily) or too infrequent (twice a day versus 3 times a day) for a $70-\mathrm{kg}$ adult. ${ }^{7}$ Potentially low, infrequent dosing may have con- tributed to some clinical failures in the $\beta$-lactam arms of the trials in the meta-analysis.

Amoxicillin and second-generation cephalosporins are also of a much narrower spectrum compared with respiratory fluoroquinolones and are therefore less likely to cause resistance in a wide range of gut flora. For these reasons, the authors are correct in pointing out that $\beta$-lactam antibiotics continue to play an important role in the treatment of acute bacterial sinusitis caused by penicillin-susceptible strains of $S$. pneumoniae, most penicillin-resistant strains of $S$. pneumoniae and most strains of $H$. influenzae. The addition of clavulanic acid increases coverage for $\beta$-lactamase-positive $H$. influenzae and $M$. catarrhalis. Cephalosporins are adequate but are not as active against penicillin-resistant strains of $S$. pneumoniae.

Each year, sinusitis affects I in 7 adults. It is the fifth most common reason for prescribing antibiotics. A recent Canadian study showed that the use of fluoroquinolones to treat community-acquired pneumonia in Manitoba increased from $6.6 \%$ in $1996 / 97$ to $25.2 \%$ in $2001 / 02 .{ }^{8}$ Given that clinical sinusitis is more common than pneumonia, the prescribing of fluoroquinolones for sinusitis may have an enormous impact on resistance patterns of bacteria. The role of fluoroquinolones in the treatment of acute sinusitis in special situations or treatment failures should be considered on an individual basis. Despite their allure, there are good reasons not to use fluoroquinolones at present. As Karageorgopoulos and colleagues state, these antimicrobials should not be endorsed as first-line therapy for the treatment of acute communityacquired bacterial sinusitis. Such use would have public health consequences that would far outweigh the benefit of curing a self-limited infection that can be treated with many other effective and cheaper drugs.

Competing interests: None declared.

\section{REFERENCES}

I. Rosenfeld RM, Singer M, Jones S. Systematic review of antimicrobial therapy in patients with acute rhinosinusitis. Otolaryngol Head Neck Surg 2007;137:S32-45.

2. Karageorgopoulos DE, Giannopoulou KP, Grammatikos AP, et al. Fluoroquinolones compared with $\beta$-lactam antibiotics for the treatment of acute bacterial sinusitis: a meta-analysis of randomized controlled trials. CMAJ 2008;178:845-54.

3. Fuller JD, Low DE. A review of Streptococcus pneumoniae infection treatment failures associated with fluoroquinolone resistance. Clin Infect Dis 2005;4I:II8-2I.

4. Powis J, McGeer A, Green K, et al. In vitro antimicrobial susceptibilities of Streptococcus pneumoniae clinical isolates obtained in Canada in 2002. Antimicrob Agents Chemother 2004;48:3305-II.

5. Pletz MWR, McGee L, Jorgensen J, et al. Levofloxacin-resistant invasive Streptococcus pneumoniae in theUnited States: evidence for clonal spread and the impact of conjugate vaccine. Antimicrob Agents Chemother 2004;48:349I-7.

6. Deshpande LM, Sader HS, Debbia E, et al. Emergence and epidemiology of fluoroquinolone-resistant Streptococcus pneumoniae strains from Italy: report from the SENTRY Antimicrobial Surveillance Program (200I-2004). Diagn Microbiol Infect Dis 2006;54:157-64.

7. Jacobs MR. Optimisation of antimicrobial therapy using pharmacokinetic and pharmacodynamic parameters. Clin Microbiol Infect 200I;7:589-96.

8. Carrie AG, Kozyrskyj AL. Outpatient treatment of community-acquired pneumonia: evolving trends and a focus on fluoroquinolones. Can J Clin Pharmacol 2006; I3:eIO2-II.

Correspondence to: Dr. Nicole Le Saux, Department of Infectious Diseases, Children's Hospital of Eastern Ontario, 40I Smyth Rd., Ottawa ON KIH 8LI; fax 6I3 738-4832; lesaux@cheo.on.ca 